\title{
Dynamical Symmetry Breaking and Phase Transitions in Driven Diffusive Systems
}

\author{
Yongjoo Baek, ${ }^{1, *}$ Yariv Kafri, ${ }^{1}$ and Vivien Lecomte ${ }^{2,3}$ \\ ${ }^{1}$ Department of Physics, Technion, Haifa 32000, Israel \\ ${ }^{2}$ LIPhy, Université Grenoble Alpes and CNRS, F-38042 Grenoble, France \\ ${ }^{3}$ Laboratoire Probabilités et Modèles Aléatoires, UMR7599 CNRS, Sorbonne Paris Cité, \\ Université Pierre et Marie Curie and Université Paris Diderot, F-75013 Paris, France
}

(Received 21 September 2016; published 20 January 2017)

\begin{abstract}
We study the probability distribution of a current flowing through a diffusive system connected to a pair of reservoirs at its two ends. Sufficient conditions for the occurrence of a host of possible phase transitions both in and out of equilibrium are derived. These transitions manifest themselves as singularities in the large deviation function, resulting in enhanced current fluctuations. Microscopic models which implement each of the scenarios are presented, with possible experimental realizations. Depending on the model, the singularity is associated either with a particle-hole symmetry breaking, which leads to a continuous transition, or in the absence of the symmetry with a first-order phase transition. An exact Landau theory which captures the different singular behaviors is derived.
\end{abstract}

DOI: 10.1103/PhysRevLett.118.030604

In recent years there has been much activity focused on understanding probability distributions in systems which are far from thermal equilibrium. Specifically, the probability of observing a current flowing between two reservoirs, through an interacting channel, was studied in many works for both quantum [1-5] (in the context of "full counting statistics") and classical systems [6-35]. The properties of the distribution encode much information about the interactions in the channel.

One of the most dramatic consequences of such interactions is the occurrence of dynamical phase transitions (DPTs) [10,11,13,35-41], which are the focus of this Letter [42]. They imply an enhanced probability of observing certain current fluctuations. Beyond certain current thresholds, the mode of transport through the channel changes abruptly. These DPTs manifest themselves as singularities in a large deviation function (LDF) that characterizes the probability distribution of the timeaveraged current $J$ in the limit of a large observation time. The function plays, for time-integrated observables like $J$, the same role as the equilibrium free energy for static observables [43]. For classical interacting particle systems, it can be computed using exact microscopic solutions [6-8,14,17,21,22,24,26,32] or macroscopic approaches (see Ref. [44] for a review).

To date, for current large deviations in driven diffusive systems, only one class of DPTs with concrete microscopic models has been observed; these occur solely for periodic systems which are not connected to reservoirs $[10,11,13,15,41]$. There one finds that, for currents close to the mean value, the fluctuation manifests itself through a time-independent density profile. The DPT occurs at a critical value of the current beyond which the fluctuation is realized through a time-dependent density profile. Such transitions are referred to as resulting from a failure of the "additivity principle" [9]. Another scenario which involves a "first-order" transition between two distinct timeindependent density profiles was suggested in Ref. [12]. However, lacking any concrete microscopic models, the scenario remains speculative.

In this Letter we study current large deviations in onedimensional diffusive systems coupled to two reservoirs. Based on an exact Landau theory for the DPTs derived using the macroscopic fluctuation theory [44,45], we obtain the following new results: first, we identify DPTs between timeindependent density profiles, along with sufficient conditions for their existence in terms of transport coefficients; second, we describe a new type of "second-order" DPTs associated with a symmetry breaking in the density profiles which realize the current fluctuations; third, we show that wellstudied microscopic models-namely, the Katz-LebowitzSpohn (KLS) [46] model and the weakly asymmetric simple exclusion process (WASEP) [47,48]—implement both the first- and second-order DPTs described above; finally, possible experimental realizations are discussed.

Settings.-We consider a one-dimensional driven diffusive system connecting two particle reservoirs using the standard approach of fluctuating hydrodynamics $[4,44,49,50]$. The particle density profile $\rho(x, t)$ evolves according to a continuity equation,

$$
\partial_{t} \rho(x, t)+\partial_{x} j(x, t)=0,
$$

where the spatial coordinate $x$ is rescaled by the system size $L$ so that $x \in[0,1], t$ denotes the time measured in units of $L^{2}$, and $j(x, t)$ is the fluctuating current given by

$$
j(x, t)=-D(\rho) \partial_{x} \rho+\sigma(\rho) E+\sqrt{\sigma(\rho)} \eta(x, t) .
$$


The current consists of contributions from Fick's law, the response to a bulk field $E$, and a noise term. The diffusivity $D(\rho)$ and the mobility $\sigma(\rho)$ are, in general, density dependent and are connected by the Einstein relation, $2 D(\rho) / \sigma(\rho)=\partial_{\rho}^{2} f(\rho)$, with $f(\rho)$ being the free energy density of the system at equilibrium. The noise $\eta(x, t)$ satisfies $\langle\eta(x, t)\rangle=0$ and

$$
\left\langle\eta(x, t) \eta\left(x^{\prime}, t^{\prime}\right)\right\rangle=L^{-1} \delta\left(x-x^{\prime}\right) \delta\left(t-t^{\prime}\right),
$$

where $\langle\cdot\rangle$ denotes an average over all realizations of the noise. The spatial boundary conditions are fixed as $\rho(0, t)=\bar{\rho}_{a}$ and $\rho(1, t)=\bar{\rho}_{b}$, where $\bar{\rho}_{a}$ and $\bar{\rho}_{b}$ are timeindependent densities imposed by the reservoirs. We are interested in phase transitions $[10,11,13]$ associated with the time-averaged current

$$
J \equiv \frac{1}{T} \int_{0}^{T} d t \int_{0}^{1} d x j(x, t),
$$

whose statistics obey a large deviation principle [44]

$$
P(J) \sim \exp [-T L \Phi(J)]
$$

for $T \gg 1$. A singularity in the LDF $\Phi(J)$ marks a DPT. It proves to be convenient to change ensembles and work with the scaled cumulant generating function (CGF)

$$
\Psi(\lambda) \equiv \lim _{T \rightarrow \infty} \frac{1}{T L} \ln \left\langle e^{T L \lambda J}\right\rangle .
$$

Standard saddle-point arguments [43] show that the scaled CGF is related to the LDF by a Legendre transform $\Psi(\lambda)=\sup _{J}[\lambda J-\Phi(J)]$. To calculate $\Psi(\lambda)$, we rewrite Eq. (6) in a path integral form using the Martin-Siggia-Rose formalism [51], which gives

$$
\Psi(\lambda)=\lim _{T \rightarrow \infty} \frac{1}{T L} \ln \int \mathcal{D} \rho \mathcal{D} \hat{\rho} e^{-L \int_{0}^{T} d t \int_{0}^{1} d x\left[\hat{\rho} \partial_{t} \rho-H(\rho, \hat{\rho})\right]},
$$

with the Hamiltonian density $H(\rho, \hat{\rho})$ defined as

$$
H(\rho, \hat{\rho}) \equiv-D(\rho)\left(\partial_{x} \rho\right)\left(\partial_{x} \hat{\rho}\right)+\frac{\sigma(\rho)}{2}\left(\partial_{x} \hat{\rho}\right)\left(2 E+\partial_{x} \hat{\rho}\right) .
$$

The "momentum" variable $\hat{\rho}$ satisfies the boundary conditions (see the Supplemental Material [52]) $\hat{\rho}(0)=0$ and $\hat{\rho}(1)=\lambda$. The scaled CGF $\Psi(\lambda)$ can then be obtained using a saddle-point method. For our cases of interest, we argue that the saddle-point solutions are time independent before and after the transition. The calculations are detailed in the Supplemental Material [52] and yield profiles $\rho^{*}(x)$ and $\hat{\rho}^{*}(x)$, which minimize the action $\int_{0}^{T} d t \int_{0}^{1} d x\left[\hat{\rho} \partial_{t} \rho-\right.$ $H(\rho, \hat{\rho})]$. These profiles, which are called the optimal profiles, represent the dominant realizations of current fluctuations at a given value of $\lambda$. As we will see, phase transitions are associated with abrupt changes in the shape of the optimal profile as $\lambda$ is varied.

Results. - In what follows, we first consider systems with the equal boundary densities $\bar{\rho}_{a}=\bar{\rho}_{b}=\bar{\rho}$, with $\bar{\rho}$ very close to an extremum of $\sigma(\rho)$. Already in this case, depending on $D(\rho)$ and $\sigma(\rho)$, all of the singular behaviors described above are observed. Interestingly, this includes systems which are in equilibrium. Then, for more general boundary conditions given by $\bar{\rho}_{a}=\bar{\rho}-\delta \rho$ and $\bar{\rho}_{b}=\bar{\rho}+\delta \rho$, we argue perturbatively to the leading order in $\delta \rho$ that the behaviors are unchanged up to a shift of the transition point.

As shown in the Supplemental Material [52], the problem of minimizing over profiles can be reexpressed as

$$
\Psi(\lambda)=\frac{\bar{\sigma}}{2} \lambda(\lambda+2 E)-\inf _{m} \mathcal{L}(m),
$$

where the Landau-like function $\mathcal{L}(m)$ of the parameter $m \in \mathbb{R}$, which captures the singular behaviors of $\Psi(\lambda)$, can be written in a truncated form,

$$
\begin{aligned}
\mathcal{L}(m) \simeq & -\frac{2 \pi \bar{D}^{2}}{\bar{\sigma} \bar{\sigma}^{\prime \prime}} \bar{\sigma}^{\prime} m-\frac{\left(\lambda_{c}+E\right) \bar{\sigma}^{\prime \prime}}{4}\left(\lambda-\lambda_{c}\right) m^{2} \\
& -\frac{2 \pi \bar{D}\left(\bar{D} \bar{\sigma}^{(3)}-3 \bar{D}^{\prime} \bar{\sigma}^{\prime \prime}\right)}{9 \bar{\sigma} \bar{\sigma}^{\prime \prime}} m^{3} \\
& +\left(\frac{\pi^{2} \bar{D}\left(4 \bar{D}^{\prime \prime} \bar{\sigma}^{\prime \prime}-\bar{D} \bar{\sigma}^{(4)}\right)}{64 \bar{\sigma} \bar{\sigma}^{\prime \prime}}+\frac{\bar{\sigma}^{\prime \prime 2} E^{2}}{64 \bar{\sigma}}\right) m^{4} .
\end{aligned}
$$

Here, $\lambda_{c}$ is equal to one of the two values [56]

$$
\lambda_{c}^{ \pm} \equiv-E \pm \sqrt{E^{2}+\frac{2 \pi^{2} \bar{D}^{2}}{\bar{\sigma} \bar{\sigma}^{\prime \prime}}},
$$

and we use the shorthand notations $\bar{g} \equiv g(\bar{\rho}), \bar{g}^{\prime} \equiv g^{\prime}(\bar{\rho})$, $\bar{g}^{\prime \prime} \equiv g^{\prime \prime}(\bar{\rho})$, and $\bar{g}^{(n)} \equiv g^{(n)}(\bar{\rho})$ for derivatives of any function $g(\rho)$ evaluated at $\rho=\bar{\rho}$. The optimal value of the order parameter $m$ in Eq. (9), which we denote by $m^{*}$, measures the deviation of the optimal profile from the flat reference profile of density $\bar{\rho}$ (similar to the zero magnetization in the Landau theory for the Ising model):

$$
\rho^{*}(x)=\bar{\rho}+m^{*} \sin (\pi x)+O\left[\left(m^{*}\right)^{2}\right] .
$$

The scaled CGF $\Psi(\lambda)$ has a singularity when $m^{*}$ changes in a singular manner as $\lambda$ is varied [57].

Clearly, $\mathcal{L}(m)$ can be truncated as in Eq. (10) only if the coefficient of $m^{4}$ is positive. For the microscopic models we study below, this is always the case. While there could be other models for which higher-order terms in $m$ need to be considered, these are beyond the scope of this Letter. Moreover, for a transition to occur as $\lambda$ is varied, we need $\bar{\sigma}^{\prime}=0$, and the $\lambda_{c}$ defined in Eq. (11) has to be real valued. This is the case if $\sigma(\rho)$ has a local minimum at $\rho=\bar{\rho}$, so 
that $\bar{\sigma}^{\prime \prime}>0$; otherwise, if $\bar{\sigma}^{\prime \prime}<0$, the bulk field has to be sufficiently strong so that

$$
E^{2}>\frac{2 \pi^{2} \bar{D}^{2}}{\bar{\sigma}\left|\bar{\sigma}^{\prime \prime}\right|} .
$$

We observe different transition behaviors depending on the sign of $\bar{\sigma}^{\prime \prime}$, each of which we discuss in the following.

Case 1a: $\bar{\sigma}^{\prime \prime}>0$, symmetry breaking.-Consider a particle-hole symmetric system whose Hamiltonian density, shown in Eq. (8), is invariant under the transformation defined by $x \rightarrow 1-x, \quad \rho(x, t) \rightarrow 2 \bar{\rho}-\rho(1-x, t)$, and $\hat{\rho}(x, t) \rightarrow \lambda-\hat{\rho}(1-x, t)$. Assuming that $D(\rho)$ and $\sigma(\rho)$ are analytic, their odd-order derivatives vanish at $\rho=\bar{\rho}$, i.e., $\bar{D}^{(2 n+1)}=\bar{\sigma}^{(2 n+1)}=0$ for $n=0,1, \ldots$. Then only the $m^{2}$ and $m^{4}$ terms survive in Eq. (10), turning $\mathcal{L}(m)$ into the form of a Landau free energy of Ising-like systems. For $\lambda_{c}^{-}<\lambda<\lambda_{c}^{+}, \mathcal{L}(m)$ is minimized at $m^{*}=0$, and $\Psi(\lambda)$ has a quadratic form corresponding to Gaussian fluctuations. For $\lambda>\lambda_{c}^{+}$or $\lambda<\lambda_{c}^{-}$, we have $m^{*} \sim \pm\left|\lambda-\lambda_{c}\right|^{1 / 2}$, corresponding to a pair of symmetry-breaking profiles given by Eq. (12) which are mutually related by a particle-hole transformation defined above. This implies that, for each instance of a current fluctuation $J$ in this regime, there is a symmetry breaking so that one of the two optimal profiles is observed with equal probability (see Fig. 1). Near the transition points, the scaled CGF $\Psi(\lambda)$ has singularities which behave as $\lim _{\lambda \downarrow \lambda_{c}} \Psi(\lambda)-\lim _{\lambda \uparrow \lambda_{c}} \Psi(\lambda) \sim\left|\lambda-\lambda_{c}\right|^{2}$, implying second-order transitions. Clearly, the same critical scaling behavior is observed if $\bar{D}^{(3)}, \bar{\sigma}^{(5)}$ or higher-order derivatives are nonzero, although in such cases only one of the two density profiles is optimal.

Case 1b: $\bar{\sigma}^{\prime \prime}>0$, first-order transition.-Now consider the case where $\bar{D}^{\prime}$ and $\bar{\sigma}^{(3)}$ have nonzero values. For a consistent Landau theory, we assume that $\bar{D}^{\prime}$ and $\bar{\sigma}^{(3)}$ scale as $m^{*}$. Then the $\mathrm{m}^{3}$ term induces a weak first-order singularity of the scaled CGF [58]. On general grounds, similar results will be obtained even if $\bar{D}^{\prime}$ and $\bar{\sigma}^{(3)}$ are larger. The transition shows up as jumps of $m^{*}$ at transition points $\lambda_{d}^{ \pm}$, which are slightly shifted from $\lambda_{c}^{ \pm}$, respectively (see the Supplemental Material [52]). In a manner similar to case 1a, the fluctuations are Gaussian for $\lambda_{d}^{-}<\lambda<\lambda_{d}^{+}$, and non-Gaussian otherwise (see Fig. 1). This behavior corresponds to a scenario discussed in Ref. [12]: when a current fluctuation $J$ occurs within the intervals $\left[J_{1}^{ \pm}, J_{2}^{ \pm}\right]$defined by $J_{1}^{ \pm} \equiv \lim _{\lambda \uparrow \lambda_{d}^{ \pm}} \Psi^{\prime}(\lambda)$ and $J_{2}^{ \pm} \equiv \lim _{\lambda \downarrow \lambda_{d}^{ \pm}} \Psi^{\prime}(\lambda)$, we observe $J_{1}^{ \pm}$and $J_{2}^{ \pm}$, with probabilities $p_{1}^{ \pm}$and $1-p_{1}^{ \pm}$, respectively, such that $J=p_{1}^{ \pm} J_{1}^{ \pm}+\left(1-p_{1}^{ \pm}\right) J_{2}^{ \pm}$. This is a direct analog of phase coexistence in equilibrium first-order transitions.

Case 2: $\bar{\sigma}^{\prime \prime}<0$. - For case 1, the bulk field $E$ is not essential for the existence of a DPT: it only shifts the location of the transition point according to Eq. (11). By contrast, for case 2 , phase transitions occur only when the bulk field $E$ is strong enough to satisfy Eq. (13). Since the form of $\mathcal{L}(m)$ remains the same, the system again exhibits symmetry-breaking transitions for fully particle-hole symmetric systems, and first-order transitions in the absence of symmetry due to nonzero $\bar{D}^{\prime}$ and $\bar{\sigma}^{(3)}$. Note that, while the regions of non-Gaussian fluctuations were unbounded in case 1 , here they are bounded. This is because, for $\bar{\sigma}^{\prime \prime}<0$, both transition points $\lambda_{c}^{ \pm}$have the same sign, as implied by Eq. (11) (see Fig. 1).

Generalization to $\rho_{a} \neq \rho_{b}$.-We now turn to the case of unequal boundary densities given by $\bar{\rho}_{a}=\bar{\rho}-\delta \rho$ and $\bar{\rho}_{b}=\bar{\rho}+\delta \rho$. Treating $\delta \rho$ as a perturbation, we find, to linear order in $\delta \rho$, that (see the Supplemental Material [52])

$$
\Psi(\lambda)=\frac{\bar{\sigma}}{2} \lambda(\lambda+2 E)-2 \delta \rho \bar{D} \lambda-\inf _{m} \mathcal{L}(m),
$$

with only the quadratic term in $\mathcal{L}(m)$ modified as

$$
\left(\lambda-\lambda_{c}\right) m^{2} \rightarrow\left(\lambda-\lambda_{c}-\frac{2 \bar{D}}{\bar{\sigma}} \delta \rho\right) m^{2},
$$
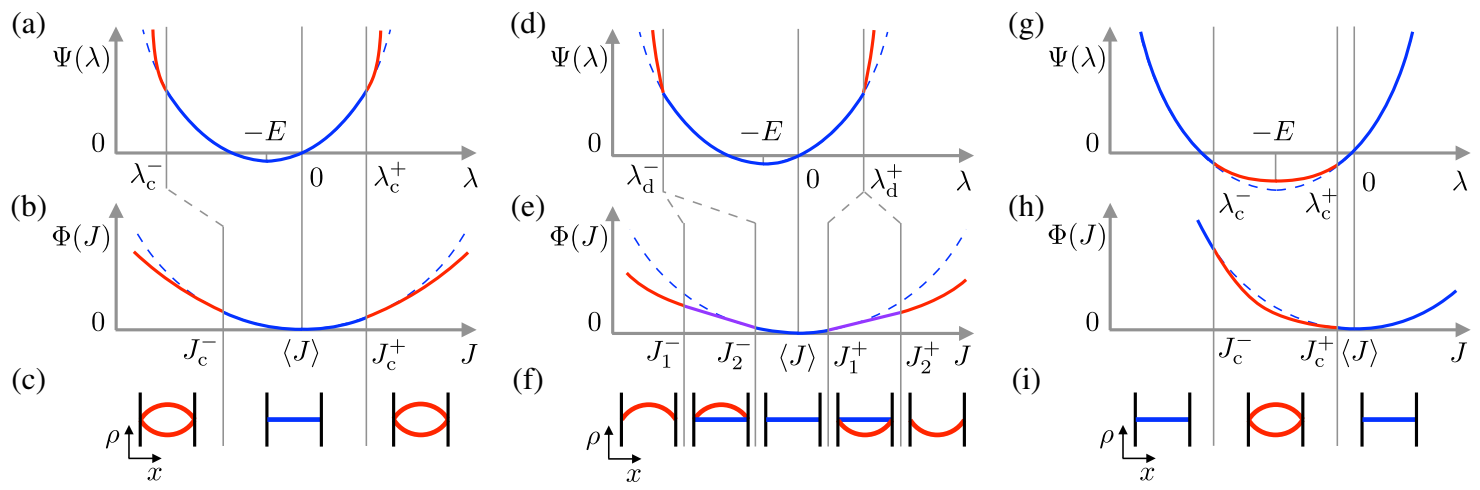

FIG. 1. Schematic illustrations of the singularities and the optimal profiles for different types of phase transitions. The dashed (blue) lines represent behaviors of the functions if the Gaussian fluctuations persist for any $\lambda$ and $J$. Case 1 . (a) The scaled CGF showing second-order singularities and (b) the corresponding LDF. (c) The shapes of optimal profiles as $J$ is varied. (d) The scaled CGF showing first-order singularities and (e) the corresponding LDF. (f) The optimal profiles as $J$ is varied. Case 2. (g) The scaled CGF showing second-order singularities and (h) the corresponding LDF. (i) The optimal profiles as $J$ is varied. 
which implies that the transition point is shifted but the other properties of the phase transitions are unchanged. If $\bar{\rho}_{a}-\bar{\rho} \neq \bar{\rho}-\bar{\rho}_{b}$, we can use $\left(\bar{\rho}_{a}+\bar{\rho}_{b}\right) / 2$ as the new value of $\bar{\rho}$. Provided that the odd-order derivatives of $D(\rho)$ and $\sigma(\rho)$ evaluated at the new $\bar{\rho}$ remain small, all of the results presented above are still valid.

Microscopic models.-We now present two lattice gas models, each of which exhibits one of the two cases of phase transitions described above.

Case 1: $\bar{\sigma}^{\prime \prime}>0$.-We consider a KLS [46] model with zero bulk bias, which features on-site exclusion and nearest-neighbor interactions. It is defined on a onedimensional lattice, each site of which can be either occupied (1) or empty (0). The model is characterized by the two parameters $\delta$ and $\varepsilon$, which govern the hopping dynamics according to the following transition rates (in arbitrary units):

$$
\begin{array}{ll}
0100 \stackrel{1+\delta}{\longrightarrow} 0010, & 1101 \stackrel{1-\delta}{\longrightarrow} 1011, \\
1100 \stackrel{1+\varepsilon}{\longrightarrow} 1010, & 1010 \stackrel{1-\varepsilon}{\longrightarrow} 0110 .
\end{array}
$$

Spatially inverted versions of these transitions occur with identical rates. Using the methods of Refs. [50,59,60], $D(\rho)$ and $\sigma(\rho)$ of the model can be derived exactly as functions of $\rho \in[0,1]$ (see the Supplemental Material [52] for their explicit forms). If $\delta=0$, the model possesses a particlehole symmetry, so that all odd-order derivatives of $D(\rho)$ and $\sigma(\rho)$ with respect to $\rho$ vanish at $\rho=1 / 2$. More interestingly, for $\epsilon>4 / 5$, one finds that $\sigma(1 / 2)$ becomes a local minimum. Thus, all results of case 1a can be applied to this model by setting $\bar{\rho}=1 / 2$. On the other hand, if $\delta \neq 0$, the system does not have a particle-hole symmetry. Then, for $\epsilon$ greater than some $\delta$-dependent threshold, $\sigma(\rho)$ has a local minimum at some $\delta$-dependent $\bar{\rho}$. All of the results of case $1 \mathrm{~b}$ are then applicable to this system.

Case 2: $\bar{\sigma}^{\prime \prime}<0$. - Consider a WASEP on a onedimensional lattice of $L$ sites whose hopping rates (in arbitrary units) are given by $10^{1+\delta} \rightarrow 01,01 \stackrel{1-\delta}{\rightarrow} 10$. If $\delta=E / L$, it is well known $[47,48]$ that the system is characterized by $D(\rho)=1$ and $\sigma(\rho)=2 \rho(1-\rho)$, so $\sigma^{\prime \prime}(\rho)<0$ for any $\rho \in[0,1]$, with the maximum of $\sigma(\rho)$ located at $\rho=1 / 2$. Applying the results of case 2, Eq. (13) implies that the system exhibits singularities of LDFs when $|E|>\pi$.

Mechanism for symmetry breaking.-To gain more intuition into the origin of the DPT, it is helpful to examine the Lagrangian formulation of the LDF [44]

$$
\Phi(J)=\inf _{\rho} \int_{0}^{1} d x \frac{\left[J+D(\rho) \partial_{x} \rho-\sigma(\rho) E\right]^{2}}{2 \sigma(\rho)} .
$$

Close to the transition point, $\Phi(J)$ is minimized by an optimal profile of the form $\rho(x)=\bar{\rho}+m \sin (\pi x)$. Keeping the leading-order corrections in $m$, we obtain
$\Phi(J) \simeq \frac{\delta J^{2}}{2 \bar{\sigma}}+\inf _{m}\left[\left(\frac{\bar{D}^{2}}{2}-\frac{\bar{\sigma}^{\prime \prime} E \delta J}{2}-\frac{\bar{\sigma}^{\prime \prime} \delta J^{2}}{4 \bar{\sigma}}\right) m^{2}+O\left(m^{4}\right)\right]$,

where $\delta J \equiv J-\bar{\sigma} E$. The occurrence of symmetry breaking is controlled by the sign of the coefficient in front of $\mathrm{m}^{2}$, whose three terms represent contributions from diffusion, the bulk field $E$, and the noise amplitude. The first two originate from the numerator of Eq. (16) and the last one comes from the denominator. The competition between these factors dictates whether it is beneficial to break the symmetry by density modulations. Depending on the sign of $\bar{\sigma}^{\prime \prime}$, there are two possible scenarios.

If $\bar{\sigma}^{\prime \prime}>0$, the coefficient of $m^{2}$ is positive for a $\delta J$ close to zero and becomes negative for a sufficiently large $\delta J$, signaling the symmetry-breaking transition-for a large enough $\delta J$, the gain in action from the denominator overwhelms the cost of density modulations in the numerator.

On the other hand, if $\bar{\sigma}^{\prime \prime}<0$, both the diffusion and the noise lead to a positive cost for density modulations. Negative contributions arise only from the field term. A large enough $E$ can make density modulations favorable for an intermediate range of $\delta J$, inducing a transition.

The origins of DPTs in these two cases are different. For $\bar{\sigma}^{\prime \prime}>0$, the transitions are due to the competition between the diffusion, which favors a flat profile, and the noise, which favors modulations. By contrast, for $\bar{\sigma}^{\prime \prime}<0$, the transitions are ruled by the contribution of the bulk field, which favors modulations, competing against the diffusion and the noise, both of which favor a flat profile. Similar arguments also apply to first-order transitions.

Comparisons with previous studies are in order. A recent study [33] proposed a criterion which forbids the DPTs of case 2; however, our results explicitly show that the WASEP is a counterexample to this criterion [61]. We note that the asymmetric simple exclusion process (ASEP), which is nondiffusive, also exhibits DPTs in current fluctuations $[26,32,62,63]$. While these DPTs are remnants of the well-known boundary-induced transitions in mean behaviors, the DPTs of diffusive systems discussed above are very different.

There remains the question of how the DPTs discussed so far can be experimentally observed. Recently, the LDF for heat current in an $R C$ circuit was empirically measured in Ref. [64], where the fast electronic dynamics allows the current LDF to be measured over a wide range [65]. To observe the DPTs discussed here in a similar experiment, one has to look at diffusive electronic transport with an extremum in $\sigma(\rho)$. These are common, resulting from nonmonotonic changes in the electronic density of states. For example, minima of $\sigma(\rho)$ were observed in graphene transport [66], and maxima in fullerene peapods [67]. Using these systems, both cases of DPTs discussed above can, in principle, be observed. 
In summary, we have studied a general one-dimensional diffusive transport through a channel connecting two reservoirs. Using a perturbative approach for a general $D(\rho)$ and $\sigma(\rho)$, we have found a large class of new DPTs which are not associated with the breaking of the additivity principle, in the sense that the optimal profiles remain time independent before and after the transition. For some of these DPTs we can explicitly prove the validity of the additivity principle, which we expect to hold for all cases (see Ref. [52]). It would be interesting to check on whether other kinds of DPTs occur at larger values of $J$ or $\delta \rho$, and how the results can be generalized to higher dimensions.

We are grateful to Paul Krapivsky for his collaboration at the early stages of this work. We also thank Giovanni Jona-Lasinio, Kirone Mallick, Ohad Shpielberg, Daniel Podolsky, and Michael Reznikov for their helpful comments. Y. B. and Y. K. are supported by an ISF grant, and Y. B. is supported in part at the Technion by a fellowship from the Lady Davis Foundation. V. L. is supported by the ANR-15-CE40-0020-03 Grant LSD and also acknowledges support by the ERC Starting Grant No. 680275 MALIG.

*yongjoo.baek@physics.technion.ac.il

[1] L. Levitov and G. Lesovik, JETP Lett. 58, 230 (1993).

[2] L. S. Levitov, H. Lee, and G. B. Lesovik, J. Math. Phys. (N.Y.) 37, 4845 (1996).

[3] S. Pilgram, A. N. Jordan, E. V. Sukhorukov, and M. Büttiker, Phys. Rev. Lett. 90, 206801 (2003).

[4] A. N. Jordan, E. V. Sukhorukov, and S. Pilgram, J. Math. Phys. (N.Y.) 45, 4386 (2004).

[5] C. Flindt and J. P. Garrahan, Phys. Rev. Lett. 110, 050601 (2013).

[6] B. Derrida and J. L. Lebowitz, Phys. Rev. Lett. 80, 209 (1998).

[7] B. Derrida and C. Appert, J. Stat. Phys. 94, 1 (1999).

[8] B. Derrida, B. Douçot, and P.-E. Roche, J. Stat. Phys. 115, 717 (2004).

[9] T. Bodineau and B. Derrida, Phys. Rev. Lett. 92, 180601 (2004).

[10] L. Bertini, A. De Sole, D. Gabrielli, G. Jona-Lasinio, and C. Landim, Phys. Rev. Lett. 94, 030601 (2005).

[11] T. Bodineau and B. Derrida, Phys. Rev. E 72, 066110 (2005).

[12] L. Bertini, A. De Sole, D. Gabrielli, G. Jona-Lasinio, and C. Landim, J. Stat. Phys. 123, 237 (2006).

[13] T. Bodineau and B. Derrida, C.R. Phys. 8, 540 (2007).

[14] S. Prolhac and K. Mallick, J. Phys. A 41, 175002 (2008).

[15] C. Appert-Rolland, B. Derrida, V. Lecomte, and F. van Wijland, Phys. Rev. E 78, 021122 (2008).

[16] A. Imparato, V. Lecomte, and F. van Wijland, Phys. Rev. E 80, 011131 (2009).

[17] S. Prolhac and K. Mallick, J. Phys. A 42, 175001 (2009).

[18] Y. Baek, Y. Kafri, and V. Lecomte, J. Stat. Mech. (2016) 053203.

[19] P. I. Hurtado and P. L. Garrido, Phys. Rev. Lett. 102, 250601 (2009).
[20] A. Prados, A. Lasanta, and P. I. Hurtado, Phys. Rev. Lett. 107, 140601 (2011)

[21] J. de Gier and F. H. L. Essler, Phys. Rev. Lett. 107, 010602 (2011).

[22] A. Lazarescu and K. Mallick, J. Phys. A 44, 315001 (2011).

[23] P. I. Hurtado and P. L. Garrido, Phys. Rev. Lett. 107, 180601 (2011).

[24] B. Derrida, J. Stat. Mech. (2011) P01030.

[25] M. Gorissen and C. Vanderzande, Phys. Rev. E 86, 051114 (2012).

[26] M. Gorissen, A. Lazarescu, K. Mallick, and C. Vanderzande, Phys. Rev. Lett. 109, 170601 (2012).

[27] P. L. Krapivsky and B. Meerson, Phys. Rev. E 86, 031106 (2012).

[28] B. Meerson and P. V. Sasorov, J. Stat. Mech. (2013) P12011.

[29] B. Meerson and P. V. Sasorov, Phys. Rev. E 89, 010101 (2014).

[30] M. Žnidarič, Phys. Rev. Lett. 112, 040602 (2014).

[31] P. I. Hurtado, C. P. Espigares, J. J. del Pozo, and P. L. Garrido, J. Stat. Phys. 154, 214 (2014).

[32] A. Lazarescu, J. Phys. A 48, 503001 (2015).

[33] O. Shpielberg and E. Akkermans, Phys. Rev. Lett. 116, 240603 (2016).

[34] L. Zarfaty and B. Meerson, J. Stat. Mech. (2016) 033304.

[35] O. Hirschberg, D. Mukamel, and G. M. Schütz, J. Stat. Mech. (2015) P11023.

[36] B. Derrida, J. L. Lebowitz, and E. R. Speer, Phys. Rev. Lett. 89, 030601 (2002).

[37] V. Lecomte, C. Appert-Rolland, and F. Wijland, J. Stat. Phys. 127, 51 (2007).

[38] J. P. Garrahan, R. L. Jack, V. Lecomte, E. Pitard, K. van Duijvendijk, and F. van Wijland, Phys. Rev. Lett. 98, 195702 (2007).

[39] G. Bunin, Y. Kafri, and D. Podolsky, J. Stat. Mech. (2012) L10001.

[40] Y. Baek and Y. Kafri, J. Stat. Mech. (2015) P08026.

[41] N. Tizón-Escamilla, C. Pérez-Espigares, P. L. Garrido, and P. I. Hurtado, arXiv:1606.07507.

[42] We also note that there are DPTs associated with changes of mean behaviors as boundary conditions are varied, most notably those of the ASEP (see [R. A. Blythe and M. R. Evans, J. Phys. A 40, R333 (2007)] for a review). These are phenomena of a very different origin.

[43] H. Touchette, Phys. Rep. 478, 1 (2009).

[44] L. Bertini, A. De Sole, D. Gabrielli, G. Jona-Lasinio, and C. Landim, Rev. Mod. Phys. 87, 593 (2015).

[45] L. Bertini, A. De Sole, D. Gabrielli, G. Jona-Lasinio, and C. Landim, Phys. Rev. Lett. 87, 040601 (2001); J. Stat. Phys. 107, 635 (2002); 116, 831 (2004); J. Stat. Mech. (2007) P07014.

[46] S. Katz, J. L. Lebowitz, and H. Spohn, J. Stat. Phys. 34, 497 (1984).

[47] A. De Masi, E. Presutti, and E. Scacciatelli, Ann. Inst. Henri Poincaré, B 25, 1 (1989).

[48] J. Gärtner, Stoch. Proc. Appl. 27, 233 (1987).

[49] H. Spohn, J. Phys. A 16, 4275 (1983).

[50] H. Spohn, Large Scale Dynamics of Interacting Particles (Springer, New York, 1991).

[51] P. C. Martin, E. D. Siggia, and H. A. Rose, Phys. Rev. A 8, 423 (1973); C. de Dominicis, J. Phys. (Paris), Colloq. 37, C1-247 (1976); H.-K. Janssen, Z. Phys. B 23, 377 (1976). 
[52] See Supplemental Material at http://link.aps.org/ supplemental/10.1103/PhysRevLett.118.030604, which includes Refs. [53-55], for more detailed discussions about the saddle-point equations, the Landau theory, the microscopic models, and the additivity principle.

[53] J. Tailleur, J. Kurchan, and V. Lecomte, J. Phys. A 41, 505001 (2008).

[54] G. Gallavotti and E. G. D. Cohen, Phys. Rev. Lett. 74, 2694 (1995); J. Stat. Phys. 80, 931 (1995).

[55] P. L. Krapivsky, J. Stat. Mech. (2013) P06012.

[56] This expression for $\lambda_{c}$ is consistent with Ref. [16], in which a condition for $\lambda_{c}$ was derived for the special case of constant $D(\rho)$, quadratic $\sigma(\rho)$, and $E=0$.

[57] We note that a similar Landau theory for DPTs in periodic systems was constructed in Ref. [13].

[58] An exception could occur if the system is fine-tuned to satisfy $\bar{D} \bar{\sigma}^{(3)}=3 \bar{D}^{\prime} \bar{\sigma}^{\prime \prime}$; see the Supplemental Material [52].

[59] J. S. Hager, J. Krug, V. Popkov, and G. M. Schütz, Phys. Rev. E 63, 056110 (2001).

[60] P. L. Krapivsky (unpublished).

[61] The argument for the criterion proposed by Ref. [33] does not apply to the case when $\left|\partial_{x} \hat{\rho}+E\right|<|E|$, which is true near the DPTs of case 2. See Sec. V of the Supplemental Material [52] for more details.

[62] M. Dudzinski and G. M. Schütz, J. Phys. A 33, 8351 (2000).

[63] J. de Gier and F. H. L. Essler, Phys. Rev. Lett. 95, 240601 (2005).

[64] S. Ciliberto, A. Imparato, A. Naert, and M. Tanase, Phys. Rev. Lett. 110, 180601 (2013); J. Stat. Mech. (2013) P12014.

[65] Unlike the continuous media studied here, the systems studied in Ref. [64] are described by only a few degrees of freedom. Even for such finite-dimensional systems, DPTs similar to those described in this Letter can still occur, as will be discussed in [Y. Baek, Y. Kafri, and V. Lecomte (unpublished)].

[66] S. Adam, E. H. Hwang, V. M. Galitski, and S. Das Sarma, Proc. Natl. Acad. Sci. U.S.A. 104, 18392 (2007); Y. W. Tan, Y. Zhang, K. Bolotin, Y. Zhao, S. Adam, E. H. Hwang, S. Das Sarma, H. L. Stormer, and P. Kim, Phys. Rev. Lett. 99, 246803 (2007); J. H. Chen, C. Jang, S. Adam, M. S. Fuhrer, E. D. Williams, and M. Ishigami, Nat. Phys. 4, 377 (2008).

[67] P. Utko, R. Ferone, I. V. Krive, R. I. Shekhter, M. Jonson, M. Monthioux, L. Noé, and J. Nygård, Nat. Commun. 1, 37 (2010). 